\title{
Family Presence for Patients and Separated Relatives During COVID-19: Physical, Virtual, and Surrogate
}

\author{
Teck Chuan Voo (iD) - Mathavi Senguttuvan • \\ Clarence C. Tam
}

Received: 10 May 2020 / Accepted: 3 August 2020

(C) Journal of Bioethical Inquiry Pty Ltd. 2020

\begin{abstract}
During an outbreak or pandemic involving a novel disease such as COVID-19, infected persons may need to undergo strict medical isolation and be separated from their families for public health reasons. Such a practice raises various ethical questions, the characteristics of which are heightened by uncertainties such as mode of transmission and increasingly scarce healthcare resources. For example, under what circumstances should non-infected parents be allowed to stay with their infected children in an isolation facility? This paper will examine ethical issues with three modes of "family presence" or "being there or with" a separated family member during the current COVID-19 pandemic: physical, virtual, and surrogate. Physical visits, stays, or care by family members in isolation facilities are usually prohibited, discouraged, or limited to exceptional circumstances. Virtual presence for isolated patients is often recommended and used to enable communication. When visits are disallowed, frontline workers some-
\end{abstract}

T. C. Voo $(\bowtie) \cdot$ M. Senguttuvan

Centre for Biomedical Ethics, Yong Loo Lin School of Medicine, National University of Singapore, Block MD11, Clinical Research Centre, \#02-03, 10 Medical Drive, Singapore 117597, Singapore e-mail: medvtc@nus.edu.sg

C. C. Tam

Saw Swee Hock School of Public Health, National University of Singapore and National University Health System, Tahir

Foundation Building, 12 Science Drive 2, \#10-01,

Singapore 117549, Singapore times act as surrogate family for patients, such as performing bedside vigils for dying patients. Drawing on lessons from past outbreaks such as the 2002-2003 SARS epidemic and the recent Ebola epidemic in West Africa, we consider the ethical management of these modes of family presence and argue for the promotion of physical presence under some conditions.

Keywords COVID-19 · Family presence $\cdot$ Medical isolation · Family separation · Communication .

Epidemics · Outbreaks

Public health responses to epidemics involving a novel, highly-infectious, and potentially deadly pathogen such as SARS-CoV-2 (which causes COVID-19) typically include medical isolation of infected patients to prevent and contain spread. ${ }^{1}$ These measures raise complex issues relating to family separation. For example, under what circumstances should uninfected parents be allowed to stay with their infected children in an isolation facility? Should relatives be allowed to see a dying patient for possibly one last time when routine visitation has been suspended? Many COVID-19 patients in affected countries have died alone in isolation facilities (Horowitz and Bubola 2020), as happened during past epidemics of Severe Acute Respiratory Syndrome (SARS) in 2002-2003 (Pang 2013), Middle East

\footnotetext{
${ }^{1}$ Strict medical isolation may also be used to contain infectious diseases that are not endemic to a setting such as drug-resistant tuberculosis in-non-outbreak situations.
} 
Respiratory Syndrome (MERS) in South Korea in 2015 (Shin and Nam 2015), and Ebola virus disease (EVD) in West Africa in 2014-2016 (World Health Organization 2014). Families of deceased patients are dealt a double tragedy when the bodies are disposed of immediately without funeral rites (Goh 2020a). Such measures are tragic but may be seen as a necessary cost of protecting public health, given the contagiousness and virulence of SARS-CoV-2, uncertainties regarding its transmission, and current lack of proven therapeutics and vaccines.

In this paper we examine ethical and practical issues with modes of "family presence" (FP) for isolated COVID-19 patients. Family presence refers to ways for family ${ }^{2}$ (or persons taking on familial roles) to be there for or with a medically isolated patient and can be physical, virtual, or surrogate. Physical FP is defined here as physical visits or care by family members within an isolation facility. Virtual FP refers to families being there for patients through non-face-to-face communication. In surrogate FP, non-relatives take on familial roles for patients in isolation. We examine the ethical management of these FP modes and argue for the promotion of physical FP under some conditions.

\section{Physical FP}

Medical isolation of COVID-19 patients in most countries currently occurs in acute hospitals or dedicated buildings with heightened infection prevention and control (IPC) measures to protect staff and non-COVID-19 patients. Restrictive visitation measures are also implemented for containment reasons; during the initial stage of the 2015 South Korean MERS outbreak, hospitals allowed families to provide bedside care, which likely fueled community spread (World Health Organization 2015). Overly liberal approaches to physical family presence - where relatives choose when and how they want to be with infected patients - can undermine outbreak management and lead to worse outcomes for society as a whole.

Nevertheless, policies preventing physical family presence deserve ethical scrutiny for three reasons. First, such policies vary between and within countries. Some healthcare facilities, particularly when overwhelmed,

\footnotetext{
2 "Family" here refers to people who are loved or intimately related to the patient and hence need not be biologically or formally (e.g. marriage) related to the patient.
}

may place an absolute ban on visitors for infected patients (Hafner 2020), while others may permit visits in exceptional cases, such as for dying patients on compassionate grounds. This raises concerns of unequal treatment of patients and their families. Second, denying visits or care by a family member may result in adverse and potentially long-term psychological effects for medically isolated patients, their families, and healthcare workers involved in their care (Chung et al. 2005; Gardner and Moallef 2015; Mak et al. 2009; Maunder et al. 2003). Third, policies prohibiting visitation may discourage patients from seeking treatment, undermining disease control. The last two reasons contributed to the modification of Ebola isolation centersbiosafety level 4 emergency units or tents - with transparent walls to allow safe patient-family contact (Devi 2018). In this way, Ebola patients are safely medically isolated but not socially disconnected: "family members pull up molded plastic chairs to the cubicles and even hold up small children to say hello" (Maliro 2018, Ф13).

\section{Virtual and Surrogate FP}

The World Health Organization (WHO)'s document "Guidance for Managing Ethical Issues in Infectious Disease Outbreaks" states that individuals placed in isolation or quarantine should be provided with "the means to communicate with their loved ones and the outside world" so as to "respect individual dignity and address the significant psychosocial burden of confinement on individuals and their loved ones" (World Health Organization 2016, 26). The recent position statement (part 2) of the Society of Critical Care Medicine on the ethics of outbreaks endorses e-communication, such as video calls between isolated patients and families, to support family-centered care (Papadimos et al. 2018). ${ }^{3}$

Despite this, virtual FP may not be implemented during COVID-19 because of resource or connectivity limitations or to protect the privacy of other isolated patients (Wakam et al. 2020). Telephone communication may be used in place of video calls but is not always satisfactory: "families may be left feeling like they didn't get to say goodbye properly" (Wakam et al., I6). E-communication can increase the burden on overstretched healthcare workers who may need to provide assistance to patients using

\footnotetext{
3 "Family-centered care" may be broadly defined as optimization of patient care that is planned around and experienced by families.
} 
communication devices which increases the workers' risks of infection (Goldstein and Weiser 2020; Rivas 2020; Wakam et al. 2020).

Additionally, healthcare workers may need to act as "surrogate" family by taking on roles usually performed by the patient's family, such as bedside vigils (Holmes 2020), relaying information, including non-medical information, for concerned relatives outside the facility ( $\mathrm{Ng} 2020),{ }^{4}$ and care-giving for isolated children separated from their parents (Koller et al. 2006; Shaw, Yun, and Patel 2020). While humane and compassionate, surrogate FP increases the psychological burden on healthcare workers.

\section{Ethical Management of FP Modes}

The above issues with the three FP modes provoke the need to consider their ethical management, to better balance patient and family interests and the interests of society vis-à-vis public health.

One way to do so is to apply public health ethical principles. These may require, for example, that public health interventions be effective and necessary to achieve public health goals, proportionate and least-infringing, while ensuring that measures are also in place to minimize harms to individuals from restrictive measures (Kass 2001). These principles justify actively supporting familial needs during epidemics, recognizing that restrictive measures such as isolation have effects on not only on affected individuals but also their family. This provides a strong argument for allowing virtual FP as a least-restrictive policy during epidemics, and investment in e-communication infrastructure should be regarded as an important aspect of pandemic preparedness and response. E-communication systems can be designed to remove reliance on healthcare workers' support and, importantly, they can be configured to "ensure that the families of patients in critical condition can be quickly contacted and brought to the patient's bedside virtually to be with them in their final moments" (Martineau 2020).

Beyond facilitating on-demand virtual FP, ecommunication systems have wider benefits for patient care $^{5}$ and infection control in hospitals. For example,

\footnotetext{
${ }^{4}$ Such a task is usually performed by a family spokesperson in routine care.

${ }^{5}$ These include meeting the specific needs of some patients, such as deaf patients by facilitating Virtual Remote Interpreting using the remote services of a sign language interpreter (Martineau 2020).
}

some U.S. hospitals have mounted bedside electronic tablets, allowing healthcare workers to monitor and communicate with COVID-19 patients remotely (Martineau 2020). This reduces demand for limited personal protective equipment (PPE) while reducing infection risk. The added burdens of a pandemic can make healthcare settings become "more hierarchical and less human" (McNamee 2004) and more risk-oriented than patient-oriented; allowing patients to e-interact with healthcare workers without masks (which would be required if interaction occurs at the bedside) and see their facial expressions can be comforting and humanizing (Martineau 2020), while also facilitating verbal communication. Where hospitals lack resources to provide e-communication devices, patients or families could be allowed to provide their own.

\section{Minors}

For some patients and families, virtual FP is likely to be insufficient to support their needs and well-being. During the 2002-2003 SARS epidemic, healthcare workers provided surrogate FP by acting as a "substitute family" for medically isolated young children (Koller et al. 2006, 5). Nevertheless, some of these children experienced psychological trauma from parental separation in an unfamiliar environment. Similar effects have been reported for isolated children during COVID-19 (Shaw, Yun, and Patel 2020). To inform FP policies, more research is needed on the psychological impact of medical isolation on children during a public health crisis, taking into account factors such as the severity of the illness and duration of isolation. Possibly, virtual FP and surrogate FP by healthcare workers could mitigate the harms of parental separation for isolated children with COVID-19, especially those who are older and accustomed to e-communication with their loved ones and friends.

Whether at least one (uninfected or asymptomatic) parent should be allowed to be admitted with the child into the isolation facility thus depends on the maturity of children and the likelihood and degree of harm due to parental separation and the benefits of physical FP (including for healthcare workers in terms of alleviating their care burden). These benefits and harms need to be weighed against the capacity of the public health infrastructure to mitigate the additional risks of physical FP. Children with medical or other circumstances that require special care may significantly benefit from the 
intimate care of a parent familiar with their needs, increasing the justification for physical FP. The WHO interim guidance dated 13 March 2020 on "Clinical management of severe acute respiratory infection (SARI) when COVID-19 disease is suspected" recommends that mothers and infants should remain together, especially immediately after birth, whether they or their infants have suspected or confirmed COVID-19 (World Health Organization 2020).

Parental autonomy over care of their children free from governmental intrusion, and to make decisions concerning their own health and risks, is another reason for justifying physical FP for children in medical isolation. While individual autonomy may justifiably be infringed to protect public health, parental autonomy should be respected in settings where SARS-CoV-2 transmission is relatively contained, there is little risk of an overwhelmed healthcare system, and risks are minimized for the designated caregiver. Other than allaying separation anxieties and distress, presence in the isolation unit enables parents to fulfil their moral commitments to their child, mitigates regret and grief should the child be lost, and maintains family integrity - the bond and resilience of family members to function and survive as a social unit through highly stressful or traumatic events, and as a whole afterwards. Onward transmission risk can be minimized by allowing only one parent (or, another relative deemed appropriate by the parent(s)) to stay with the child throughout the isolation period without leaving the facility (Goh 2020b) and requiring the parent to comply with necessary IPC measures to protect others and themselves. Parents should also be monitored for SARS-CoV-2 infection. If a parent has existing comorbidities that increase the risk of severe COVID-19 disease and intensive care, he or she should not be allowed to be admitted with the child for their own interest and to protect scarce resources during the pandemic. ${ }^{6}$

\footnotetext{
${ }^{6}$ In situations where infected parents need to be isolated and separated from their children, appropriate care arrangements such as by a willing relative and virtual FP should also be ensured for uninfected or asymptomatic minors who have to stay home or in a separate quarantine facility while their infected parents are medically isolated as part of a broader FP policy.
}

\section{Dying Patients}

The principles and values outlined above (leastrestrictive intervention, patient welfare, and family interest) also argue against absolute prohibition of visits for isolated dying patients. Death has been conceptualized as requiring "a communal response of 'accompanying' the dying" which "clarifies our sense of self, upholds us in our weakness, and facilitates the achievement of an art of dying" (Dugdale 2015, 183). While the presence of healthcare workers could contribute to a sense of community and "convey the love you [family] long to share in person" (Siddique and Marsh 2020, I16), healthcare workers recognize that neither they nor virtual FP can replace physical FP in these situations; allowing visits would relieve them of tremendous psychological burdens and promote a healthy emotional distance from their patients (Halbfinger 2020; Siddique and Marsh 2020). Significantly, allowing physical FP at the bedside of a dying patient would provide better closure than other modes of presence. Risks could be minimized by requiring and providing PPE and limiting visits of short duration to only one or two family members. In places where the epidemic is well-controlled, healthcare facilities could consider allowing a family member to be involved in giving comfort care to a COVID-19 patient as part of good end-of-life care. The family should be informed of the risks and virtual FP should be facilitated for those unable or reluctant to come, whether to protect themselves or others. Visitors should be required to comply with quarantine orders, which would not impose significant additional burden in countries and cities already under lock-down.

\section{Conclusion}

Strict medical isolation that prohibits physical FP for patients is a recurring ethical issue in outbreaks and has been implemented as a COVID-19 response. In our view, it is unnecessarily harsh for healthcare facilities to adopt absolute prohibition as a default and unchanging policy during the course of an epidemic. This includes residential care homes. The toll of "family absence" has been devastating for patients in these facilities, particularly dementia patients who rely on inperson family support (Wang et al. 2020; Devlin 2020). Close to ten thousand additional deaths among dementia patients in England and Wales have been 
reported, which appears to be linked to reduced care and social isolation following restrictive visitor policies (Devlin 2020). Physical FP should be facilitated for dementia patients whose care and welfare critically depend on it and who will not benefit much from virtual FP. Potentially, rigorous testing policies for residents, staff, and visitors could be implemented to mitigate the transmission risk of physical FP.

Whether to allow physical FP during COVID-19 and future outbreaks depends on clinical and epidemiological factors that influence infection and medical risks, available pharmaceutical countermeasures, and the resources of and strain on a healthcare system. If we accept that physical FP is necessary to mitigate the severe harms of isolation for at least some patients, and that it could reduce healthcare workers' burdens, then it is ethically justifiable for healthcare systems to allocate resources to facilitate physical FP, such as PPE and the training of family members to ensure effective use. This allocation is conditional on the assessment that physical FP would not divert resources away from healthcare workers and systems to the detriment of their effective functioning during a pandemic.

Physical FP may however be prohibited even when resources and other factors (e.g. low case numbers) are favourable for its implementationshortage of PPE might be used as a reason to resist allowing family visits when there is little risk of critical shortage (Halbfinger 2020). Much of this resistance lies in a simplistic view of epidemics spreading in populations made up of collections of individuals rather than complex social structures, and a "blinkered" focus on segregating infectious and uninfected individuals. The severe physical distancing measures implemented in response to COVID-19 have made the need to maintain social bonds starkly clear. Pandemic preparedness and response plans need to take into account the impact of epidemics on these social bonds. Consideration of the benefits and harm-reduction provided by physical and virtual FP can result in more patient- and family-centered isolation policies that are compatible with risk reduction, while respecting important individual and social values that should guide a community during a crisis.

Acknowledgments We thank the two anonymous reviewers for their valuable comments which have helped us to improve the manuscript.

\section{References}

Chung, B.P.M., T.K.S. Wong, E.S.B. Suen, and J.W.Y. Chung. 2005. SARS: Caring for patients in Hong Kong. Journal of Clinical Nursing 14(4): 510-551.

Devi, S. 2018. FRONTLINE: A new treatment facility for Ebola Virus Disease. Lancet 8(392): 2428.

Devlin, H. 2020. Extra 10,000 dementia deaths in England and Wales in April. The Guardian, 5 June. https:/www. theguardian.com/world/2020/jun/05/covid-19-causing10000-dementia-deaths-beyond-infections-research-says. Accessed June 08, 2020.

Dugdale, L.S. 2015. Conclusion. In Dying in the twenty-first century: Towards a new ethical framework for the art of dying well, edited by L.S. Dugdale, 173-191. Cambridge: The MIT Press.

Gardner, P. J., and P. Moallef. 2015. Psychological impact on SARS survivors: Critical review of the English language literature. Canadian Psychology 56(1):123-135.

Goh, S. 2020a. Caregiver allowed to stay with child hospitalised for COVID-19: KKH. Today, April 3. https://www. todayonline.com/voices/caregiver-allowed-stay-childhospitalised-covid-19-kkh. Accessed June 08, 2020.

- 2020b. No farewells, no ceremonies: China's Hubei cremates coronavirus dead. Reuters, March 30. https:/www.reuters.com/article/us-health-coronaviruschina-cremation/no-farewells-no-ceremonies-chinas-hubeicremates-coronavirus-dead-idUSKBN21G0YT. Accessed 08 Jun 2020.

Goldstein, J., and B. Weiser. 2020. "I cried multiple times": Now doctors are the ones saying goodbye. New York Times, April 13, updated April 14. https://www.nytimes.com/2020/04/13 /nyregion/coronavirus-nyc-doctors.html. Accessed June 08, 2020.

Halbfinger, D.M. 2020. "It's really a gift": Israeli hospitals let relatives say goodbye up close. The New York Times, April 20. https://www.nytimes.com/2020/04/20 /world/middleeast/israel-hospitals-relatives-dying.html. Accessed June 08, 2020.

Hafner, K. 2020. A heart-wrenching thing: Hospital bans on visits devastate families. New York Times, March 29. https://www. nytimes.com/2020/03/29/health/coronavirus-hospital-visitban.html. Accessed June 08, 2020.

Holmes, B. 2020. At the bedside of those dying "alone" from COVID-19, there is a loving presence. Courier Journal. April, 28. https://www.courier-journal. com/story/opinion/2020/04/28/coronavirus-deaths-thosewho-die-without-family-friends-arent-alone/3024895001/. Accessed June 08, 2020.

Horowitz, J., and E. Bubola. 2020. Italy's coronavirus victims face death alone, with funerals postponed. The New York Times, March 16, updated March 19. https://www.nytimes. com/2020/03/16/world/europe/italy-coronavirus-funerals. html. Accessed June 08, 2020.

Kass, N.E. 2001. An ethics framework for public health. American Journal of Public Health 91(11): 1776-1782.

Koller, D.F., D.B. Nicholas, R.S. Goldie, R. Gearing, and E.K. Selkirk. 2006. When family-centered care is challenged by infectious disease: Pediatric health care delivery during the SARS outbreaks. Qualitative Health Research 16(1): 47-60. 
Mak, I.W., C.M. Chu, P.C. Pan, M.G. Yiu, and V.L. Chan. 2009. Long-term psychiatric morbidities among SARS survivors. General Hospital Psychiatry 31(4): 318-326.

Maliro, A.K. 2018. In Congo, a new and less isolating Ebola treatment center. Medical XPress, September 10. https://medicalxpress.com/news/2018-09-congo-isolatingebola-treatment-center.html. Accessed June 08, 2020.

Martineau, P. 2020. iPads are crucial health care tools in combating COVID-19. Wired, April 8. https://www.wired. com/story/ipads-crucial-health-tools-combating-covid-19/. Accessed June 08, 2020.

Maunder, R., J. Hunter, L. Vincent et al. 2003. The immediate psychological and occupational impact of the 2003 SARS outbreak in a teaching hospital. Canadian Medical Association Journal 168(10): 1245-1251.

McNamee, J. 2004. Isolation, depersonalization and repeat trauma. Journal of Feminist Family Therapy 15(2-3): 121-125.

Ng, C. 2020. Coronavirus: Dead patient's family thankful for caring doctor's frequent updates. The Strait Times, April 1. https://www.straitstimes.com/singapore/dead-patientsfamily-thankful-for-caring-doctors-frequent-updates. Accessed June 08, 2020.

Pang, M. 2013. SARS in Singapore: Race against time to battle deadly disease. The Strait Times. March 16. https://www. straitstimes.com/singapore/sars-in-singapore-race-againsttime-to-battle-deadly-disease. Accessed June 08, 2020.

Papadimos, T.J., E.G. Marcolini, M. Hadian et al. 2018. Ethics of outbreak position statement. Part 2: Family-centered care. Critical Care Medicine 46(11): 1856-1860.

Rivas, S. 2020. Connecting COVID-19 patients with their families-Madrid, Spain. European Society of Cardiology, April 6. https://www.escardio.org/Education/COVID-19-andCardiology/connecting-covid-19-patients-and-their-familiesmadrid-spain. Accessed June 08, 2020.

Siddique, H., and S. Marsh. 2020. Coronavirus: Britons saying final goodbyes to dying relatives by videolink. The Guardian, March 24. https://www.theguardian. com/world/2020/mar/24/britons-saying-final-goodbyes-todying-relatives-by-videolink-covid-19. Accessed June 08, 2020 .
Shaw, A.Y., J.K. Yun, and N.A. Patel. 2020. Are we seeing the first of the COVID-19 orphans? The Washington Post, April 17. https://www.washingtonpost.com/opinions/2020/04/17 /how-coronavirus-is-exposing-new-kind-family-separation/. Accessed June 08, 2020.

Shin, S.S., and Y.S. Nam, 2015. MERS makes for lonely deathbeds. Korea JoongAng Daily, June 18 . https://koreajoongangdaily.joins.com/news/article/article. aspx?aid=3005521. Accessed June 08, 2020.

Wakam, G.K., J.R. Montgomery, B.E. Biesterveld, and C.S. Brown. 2020. Not dying alone-compassionate care in the Covid-19 pandemic. New England Journal of Medicine. ePub ahead of print, June 11. doi: https://doi.org/10.1056 /NEJMp2007781.

Wang, H., T. Li, P. Barbarino et al. 2020. Dementia care during COVID-19. Lancet 395(10231): 1190-1191.

World Health Organization. 2014. Ebola in Liberia: Misery and despair tempered by some good reasons for hope. In Ebola at 6 months - essays. World Health Organization. https://www. who.int/csr/disease/ebola/ebola-6-months/liberia/en/. Accessed July 13, 2020. - 2015. MERS-CoV joint mission findings discussion: Briefing notes on MERS-CoV, June 15. World Health O r g a n i zation. ht t p s : / / w w w. w ho. int/mediacentre/news/mers/briefing-notes/update-15-062015/en/. Accessed 13 Jul 2020.

_.2016. Guidance for managing ethical issues in infectious disease outbreaks. World Health Organization. https://apps. who.int/iris/handle/10665/250580. Accessed 13 Jul 2020.

. 2020. Clinical management of severe acute respiratory infection (SARI) when COVID-19 disease is suspected: Interim guidance, 13 March 2020. World Health Organization. https://apps.who.int/iris/handle/10665 /331446. Accessed 13 Jul 2020.

Publisher's note Springer Nature remains neutral with regard to jurisdictional claims in published maps and institutional affiliations. 